\title{
Effect of MobileASL on Communication Among Deaf Users
}

\author{
Joy Kim \\ Computer Science \& Engineering Computer Science \& Engineering \\ University of Washington \\ Seattle, WA 98195 USA \\ jojo0808@uw.edu \\ Jessica J. Tran \\ Electrical Engineering \\ University of Washington \\ Seattle, WA 98195 USA \\ jjtran@ee.washington.edu \\ Tressa W. Johnson \\ The Information School \\ DUB Group \\ University of Washington \\ Seattle, WA 98195 USA \\ tressaj@uw.edu \\ University of Washington \\ Seattle, WA 98195 USA \\ ladner@cs.washington.edu

\section{Eve Riskin} \\ Electrical Engineering \\ University of Washington \\ Seattle, WA 98195 USA \\ riskin@ee.washington.edu \\ Jacob O. Wobbrock \\ The Information School \\ DUB Group \\ University of Washington \\ Seattle, WA 98195 USA \\ wobbrock@uw.edu
}

Copyright is held by the author/owner(s).

CHI 2011, May 7-12, 2011, Vancouver, BC, Canada.

ACM 978-1-4503-0268-5/11/05.

\begin{abstract}
MobileASL, a software program enabling sign-language video on mobile devices over conventional U.S. cellular networks, was evaluated in a three-week field study during the summer of 2010. Through a series of interviews, questionnaires, and a focus group, we asked participants about their behavior with and perceptions of MobileASL. In addition, we used ondevice experience sampling and usage logging to observe how MobileASL was used. Initial results indicate that although participants felt that MobileASL's short battery life limited its use, participants took advantage of the mobility of the technology and used it for in-the-moment information gathering in places like buses, restaurants, and shopping areas.
\end{abstract}

\section{Keywords}

Mobile phones, video telephony, field study, American Sign Language, Deaf community

\section{ACM Classification Keywords}

H.5.1. [Information Interfaces and Presentation]:

Multimedia Information Systems-Video.

\section{General Terms}

Design, Human Factors. 


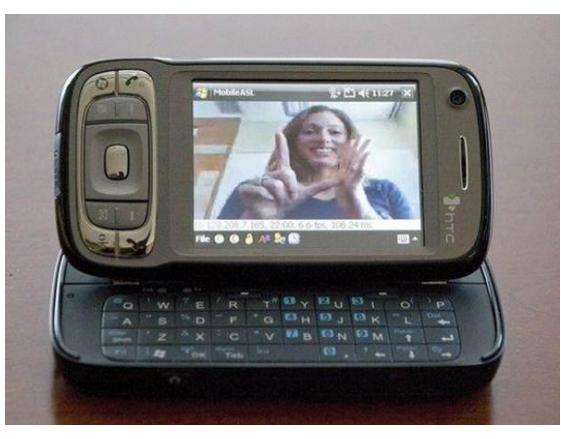

Figure 1. MobileASL running on the HTC TYTN II.

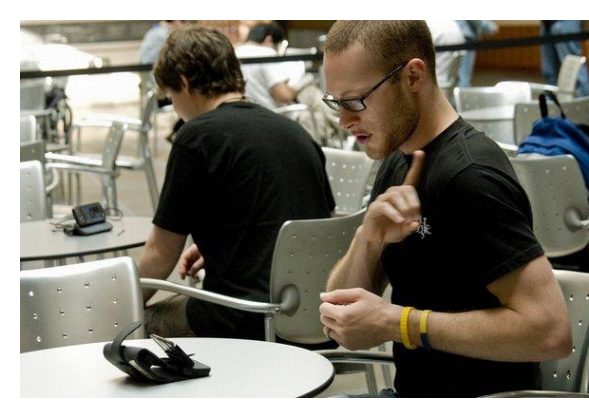

Figure 2. Two participants conversing through MobileASL.

\section{Introduction}

MobileASL [4] is a video compression project with the goal of making sign language communication over cellular networks through video a reality in the U.S. However, transmitting intelligible American Sign Language (ASL) over mobile phones is difficult due to the low bandwidth of the cellular network and the slow processor speeds on cell phones. A new real-time compression scheme is needed to transmit video within the existing wireless network while maintaining video quality that allows users to understand ASL with ease. We are designing a new ASL encoder that is compatible with the H.264/AVC [1] compression standard using the encoding software library $\times 264$ [7]. Our goal is to make ASL cell phone communication possible without the need of equipment other than a mobile phone with a front-facing camera. Figure 1 shows MobileASL running on a mobile device.

Currently, members of the Deaf community primarily use text-based means of communication (such as text messaging, instant messaging, and teletype) and/or video phones that require a setup of equipment in the home, which is not well suited to mobile use. Video cell phones would allow Deaf people to communicate in real-time using sign language. There are video chat technologies being developed for cell phones already, such as the iPhone's Facetime [2]. MobileASL, however, is unique in that it uses algorithms designed for transmitting intelligible ASL such as region-of-interest encoding around the face and hands, important areas for sign language communication.

We conducted a three-week field study deployment with 11 deaf and hard-of-hearing participants to learn how MobileASL is used in everyday life and how it influences the way Deaf people communicate. Mobile technologies are inherently meant to be used while people are on-the-go, so a field study is necessary to understand how they are used. Data collection methods included a series of interviews, questionnaires, and a focus group to ask participants about their experiences using MobileASL. To collect usage directly from the phone, we created a two-part system consisting of experience sampling [3] and unobtrusive logging. The results from this initial study will illuminate the behaviors of Deaf users of mobile phone video, and prepare us to conduct a longer-term, major field deployment in the summer of 2011.

\section{Methods}

The participants in our study were students in the 2010 Summer Academy for Advancing Deaf \& Hard of Hearing in Computing [5] at the University of Washington. This program's purpose is to allow talented deaf and hard-of-hearing college and high school students explore careers in computing. The students in this program were an ideal group of participants for our preliminary field study because they were technologically savvy and would be in regular contact with each other. Most students were at least somewhat fluent in ASL or Pidgin Signed English (PSE). Only one participant was a non-signer, and for this reason, originally opted out of participating in the study but later decided to join. There were 8 males and 3 female participants. Each student was given an HTC TyTN II cell phone equipped with MobileASL. 


\begin{tabular}{|l|l|}
\hline Method & Date \\
\hline $\begin{array}{l}\text { Pre-deployment } \\
\text { questionnaire }\end{array}$ & $7 / 28 / 2010$ \\
\hline Weekly surveys & $8 / 4 / 2010 ;$ \\
& $8 / 11 / 2010 ;$ \\
& $8 / 18 / 2010$ \\
\hline Initial interviews & $8 / 3 / 2010-$ \\
& $8 / 4 / 2010$ \\
\hline Final interviews & $8 / 18 / 2010-$ \\
& $8 / 19 / 2010$ \\
\hline Focus group & $8 / 20 / 2010$ \\
\hline
\end{tabular}

Table 1. The above table shows a timeline of questionnaires and interviews we conducted for the duration of the study.

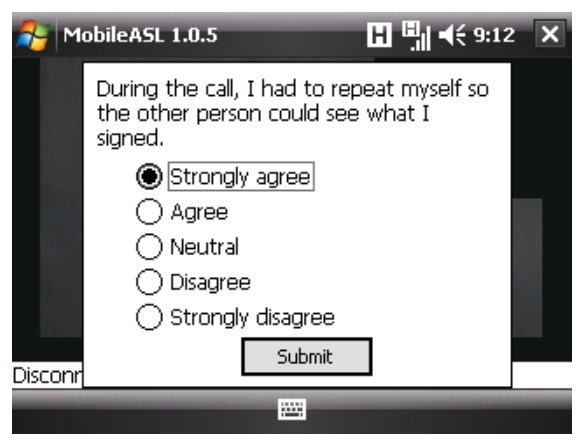

Figure 3. An experience sampling question in MobileASL.
Qualitative Research Methods

A combination of different qualitative methods was used to gather participant feedback (Table 1).

Participants took an initial questionnaire that inquired about their use of mobile and video communication technologies. A weekly survey asked the participants about the purpose of their calls, whom they called, and how useful they found MobileASL that week. During the first week of the study, participants were interviewed and asked about the methods they use to communicate and about their experiences with MobileASL. The interviews were carried out in sign language by a MobileASL researcher (a hearing, certified ASL interpreter with over 10 years of experience), recorded using video cameras, and transcribed into English by the researcher. Participants were similarly interviewed during the last week of the study, and were asked about their usage of and satisfaction with MobileASL. Unlike the first set of interviews, these interviews were carried out in spoken English by the researcher and interpreted by a professional interpreter for ease of recording. A questionnaire asking about MobileASL and video phone use was issued to participants during the study's last week. Finally, in a focus group on the final day of the study, participants discussed their experiences of MobileASL. The focus group was conducted using professional interpreters.

Technology Used in the Field Study

To collect usage data directly from participants' phones, we created two software tools: an experience sampling framework and unobtrusive logging.

\section{EXPERIENCE SAMPLING}

Experience sampling is a research method that asks participants to briefly provide a response due usually to some behavioral trigger, time event, or contextual cue [3]. When certain events occurred in MobileASL, a multiple-choice question sometimes popped up and asked the user about that event. Figure 3 is a screenshot of an experience sampling question.

We determined which events triggered questions, referring to these events as "triggers." There were six triggers used in this study; example triggers include "after a call," "after declining a call," and "change in device IP address." A server-side database held triggers, tables of questions, answer choices, and mappings among them. Each question had an equal chance of appearing for the trigger to which it was assigned. When a trigger occurred, MobileASL showed an experience sampling question if two hours had passed since the last question had been shown and the "quota" of questions to show to the user that day had not yet been met. The quota for questions in this field study was five questions per day. MobileASL showed questions more often the next day if the quota for the previous day was not met.

Users' responses were saved in XML in a text file on the phone. While MobileASL was running, it periodically uploaded the file to our server.

UNOBTRUSIVE LOGGING

During the field study, we logged information in the background about battery usage, MobileASL phone calls, changes in IP address, and how long MobileASL was kept running. No information about the content of conversations was collected. These log files were periodically sent to our server for safekeeping. 


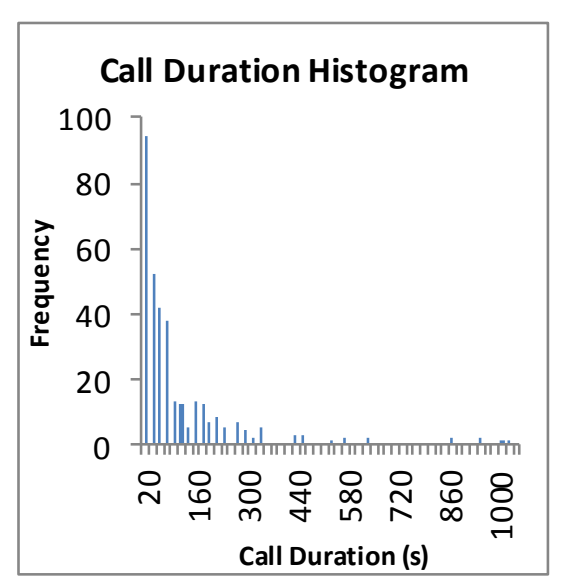

Figure 4. A histogram of the durations of calls made during the study. This does not include calls with a duration of 0 .

\section{Results}

Qualitative Results

Overall, participants expressed positive experiences using MobileASL and in many ways found the technology preferable to existing stationary videoconferencing technologies (computer video chat programs and videophones) or texting.

Through interviews, we asked participants what they liked and disliked about communication methods they already used-texting and stationary videoconferencing. Participants described texting as quick, easy to use and nearly always available. However, participants said they found it easy to misunderstand text messages. Stationary videoconferencing, however, was said to provide more cues for communication because it is visual. However, although it allows real-time sign language conversation, participants pointed out the need to be in a specific place to use it.

When asking participants about what they liked and disliked about MobileASL, they reported liking having a visual aspect to their mobile communication; not only were they able to see each other's expressions and reactions, but were able to show what they were talking about to the other person. For example, when two participants became separated from each other while shopping, they used MobileASL to show each other landmarks and eventually reconnect.

On the negative side, participants expressed that the battery life of their MobileASL devices was too short. Participants reported turning off MobileASL when they were not using it and sometimes not using MobileASL when they wanted to because it would consume too much battery life. As a result, phones were often turned off, which limited the availability of participants to each other. In addition, participants ran into occasional bad video quality. They reported dealing with this by repeating what was being said or, in one case, switching to a different mode of communication (email). Participants also pointed out that the phone was too big and the tilt of the screen needed to go further. They also mentioned wanting a touch-screen interface more easily controlled by a fingertip than a stylus.

Results from Logging and Experience Sampling Calls tended to be short, but duration varied widely ( $M=105.16 \mathrm{~s}, \mathrm{SD}=158.66 \mathrm{~s})$. Each participant typically made from 0 to 2 calls a day, except the first day of the study, where each participant made about 30 calls, and the second day, where each participant made about 7 calls (likely because of a novelty effect).

This pattern of short and occasional calls, in tandem with participants' responses to interview questions and the weekly surveys, suggests that MobileASL was used to gather information in moments of need. For example, a group of participants got lost while riding the bus to the mall. Using MobileASL, they called a participant who had remained on campus in order to ask for directions. Participants said that MobileASL was much better than texting in these cases because it would take a long time to describe the situation using text and wait for a reply. With MobileASL, participants were able to immediately receive and convey information.

Calls were mostly made from 7am - 11am and 4pm $7 \mathrm{pm}$, which were the times before and after participants' classes. This seems to suggest that although MobileASL is indeed mobile, it was not as 


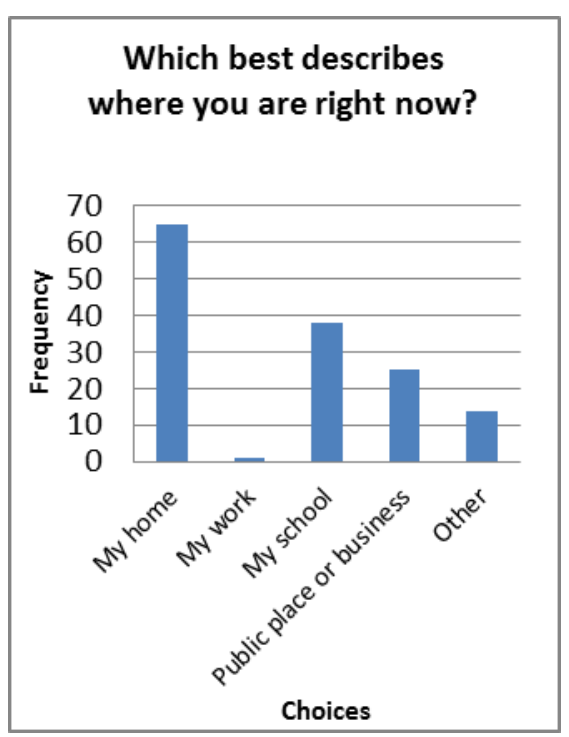

Figure 5. Distribution of responses for the experience sampling question "Which best describes where you are right now?" discreet as texting and required the full attention of users.

From experience sampling, we found that when asked about their current location (Figure 5), many responses indicated "public place or business" and "other;" participants seem to have utilized the mobility provided by MobileASL. Unfortunately, we did not receive very many responses for other experience sampling questions. Although participants were required to answer every question shown to them, participants made less calls than we anticipated, so that questions were shown less often than we intended. For the main field study, we will show questions more often.

\section{Participants' General Conclusions}

In general, although limited battery life was raised as a major issue, participants expressed overwhelmingly positive feelings while using MobileASL. The conclusion drawn by the participants can be summed up by a participant's words from the final interview:

The only thing that I don't like is of course the battery life... Other than that, everything's great... The user experience is great, it's really easy to call friends, it's easy to carry around, portable. It works in most places, unless you're of course in the mountains or something, it's faster than texting, I love that, I enjoy the ease of communication.

\section{Discussion}

Participants used MobileASL in situations that demonstrated its potential to change how the Deaf community communicates. However, as stated, short battery life on the device was pointed out as a factor that limited use. Because the device was often powered off, participants were not always available to each other for communication. Short battery life limited participants' use of MobileASL and thus our observations of how MobileASL affects communication among Deaf users. Therefore, discovering how to extend battery life to a reasonable duration is an important goal to be achieved before our main field study.

What participants liked and disliked about MobileASL overlapped with many of the general comments participants made about both texting and existing stationary videoconferencing technologies; MobileASL fulfills many of the features of both technologies. In general, participants expressed that if battery life could be extended, they would use MobileASL "all the time."

\section{Limitations}

In our preliminary study, participants were unable to call people who did not have MobileASL, such as family members. Because these interactions would be valuable to observe, we are currently building a desktop client for sending and receiving calls to and from MobileASL. We were also unable to make the issued MobileASL devices the only devices participants used for communication; it would be difficult for participants to have to tell all their contacts to switch to contacting them at a new, temporary phone number. In addition, we did not measure participants' texting use, although we plan to do so for our main field study next summer.

\section{Future Work}

In this work, we conducted a preliminary field study of MobileASL and discovered that participants found MobileASL useful and convenient. Participants seemed to use MobileASL in moments they needed information, 
and took advantage of its mobility by using it in buses, restaurants, or while travelling.

In the main field study, we will have the desktop PC version of MobileASL available. The PC version is a Windows application that uses a webcam to make calls to both the mobile and PC versions of MobileASL. This version will be distributed to family and friends of future participants so that a participant can call or be called while mobile. We also anticipate cooperating with a Video Relay Service (VRS) company to see how MobileASL can be used with VRS.

We are also implementing power-saving algorithms to extend battery life. These algorithms save power by altering the temporal and spatial resolution of transmitted video. Previously, we conducted an online survey to discover how users perceive the visual effects our algorithms have on the resulting video [6], but we would like to see how altered video and increased battery life affect MobileASL use and conversation intelligibility in a non-laboratory (field-based) setting.

We are currently porting MobileASL to the Android operating system. This new version, when completed, will address many of the problematic issues raised by participants. MobileASL on Android will be able to run on sleeker devices that provide a finger-friendly touch interface that do not require a stylus.

\section{Conclusion}

By collecting information about the non-laboratory use of MobileASL by users fluent in sign language, we hope to understand how MobileASL is used and what effects mobile video telephony has on Deaf users' communication patterns, social relationships, social coordination, use and perception of time, and sense of safety and security. The preliminary field study reported here is a first step in this direction, and produced valuable insights that will inform the larger extended field deployment in summer 2011.

\section{Acknowledgements}

We thank the staff and students of the Summer Deaf Academy for their participation and time. We also thank Jaehong Chon for his help with the MobileASL software. This work was supported in part by National Science Foundation grant IIS-0811884. Any opinions, findings, conclusions or recommendations expressed in this work are those of the authors and do not necessarily reflect those of the National Science Foundation.

\section{References}

[1] Advanced video coding for generic audiovisual services. ITU-T Recommendation H.264, March 2005.

[2] FaceTime.

http://www.apple.com/iphone/features/facetime.html.

[3] Larson, R. and Csikszentmihalyi, M. The experience sampling method. New Directions for Methodology of Social and Behavioral Science, 15 (1983), 41-56.

[4] MobileASL. http://mobileasl.cs.washington.edu/.

[5] Summer Academy for Advancing Deaf \& Hard of Hearing in Computing.

http://www.washington.edu/accesscomputing/dhh/aca demy/.

[6] Tran, J., Johnson, T., Kim, J., Rodriguez, R., Yin, S., Riskin, E., Ladner, R. and Wobbrock, J. O. A Web-Based User Survey for Evaluating Power Saving Strategies for Deaf Users of MobileASL. In Proc. ASSETS 2010, ACM Press (2010), 115-122.

[7] x264. http://developers.videolan.org/x264.html. 